\title{
An Enclosed variable focusing lens for remote welding
}

\author{
Ryoichi Kuwano ${ }^{1, a}$, Tsuyoshi Tokunaga $^{2}$, Sho Morita ${ }^{2}$, Yuta Okane ${ }^{2}$ and Yukitoshi Otani ${ }^{3}$ \\ ${ }^{1}$ Hiroshima Institute of Technology, Department of mechanical engineering systems, 2-1-1 Miyake, Saeki-ku,Hiroshima 731-5193, Japan \\ ${ }^{2}$ Chiba Institute of Technology, Precision Engineering, 2-17-1 Tsudanuma, Narashino, Chiba 275-0016, Japan \\ ${ }^{3}$ Utsunomiya University Center for Optical Research \& Education (CORE), 7-1-2 Yoto, Utsunomiya-city, Tochigi, 321-8585, Japan
}

\begin{abstract}
In this study, a variable focusing lens with a driving mechanism was developed. The surface curvature of the lens is formed via the interaction of elastic film tension and liquid pressure generated by compression of a bellows, which enables the focal length to be changed. This paper discusses the optical characteristics of the lens system. The focal length range is $250-750 \mathrm{~mm}$ with beam diameters of $0.3 \mathrm{~mm}-0.9 \mathrm{~mm}$, respectively, when a YAG laser is irradiated. In the study, the lens system was applied to laser beam welding of mild-steel sheet with a thickness of 0.5 $\mathrm{mm}$ for 3,000 shots of laser irradiation at a peak power of $4.7 \mathrm{~kW}$ and a duration of $10 \mathrm{~ms}$.
\end{abstract}

\section{Introduction}

The authors previously proposed a liquid pressurecontrolled variable focal lens $[1,2]$ whose focal length adjustability allows the control of laser energy in the optical axis direction. Other variable focal lenses have also been proposed [ $3-8]$. However, issues remain to be addressed with these lens elements; in particular, they are unsuitable for lasers with near-infrared wavelength and for high-power laser processing.

Remote welding using high-power lasers with a large focal length has been developed [9] in conjunction with the availability of high-power laser beam sources featuring high beam quality since the 1990s. Variable focal lenses are suitable for such application because the remote welding process requires frequent adjustment of positional focus.

Here the authors propose an enclosed variable focusing lens with a bellows structure. The amount of liquid in the lens cavity is kept constant. The optical characteristics of the proposed lens were compared with those of a commercially available plano-convex lens, and the use of an optical actuator for lap welding with a YAG laser was demonstrated for related application.

\section{Principles and mechanisms of variable focus}

Figure 1 outlines the principle of the proposed lens, which mainly consists of an enclosed bellows container filled with liquid on a transparent acrylic plate and a polymer film. The driving unit used to expand and contract the bellows in order to vary the shape of the refractive surface (polymer film side) is integrated with the lens. Thus, the internal pressure of the lens varies with the movement of the bellows, and the refracting surface is elastically deformed. The focal length of the lens can therefore be changed by controlling the pressure of the liquid in the lens, whose focus depends on the curvature radius of the bulging elastic spherical membrane (polymer film). The focal length of the lens is determined using a model in which the bellows are considered as a truncated cone. An outline of the bellows combined with $i$ pieces is given in Fig. 1, where $r_{1}, r_{2}$ and $h$ represent the small and large radii of the truncated cone and its height, respectively. The volume reduction that results from contracting the cone by $\Delta L \mathrm{~mm}$ in the height direction is given as $\Delta V \mathrm{~mm}^{3}$. Thus, the volume reduction $\Delta V \mathrm{~mm}^{3}$ for the entire bellows is $i \cdot \Delta V$. The ratio of $r_{1}$ to $r_{2}$, referred to as $k\left(=r_{2} / r_{1}\right)$, and the relationship between a bellows compression of $L \mathrm{~mm}$ and the result in volume reduction $V \mathrm{~mm}^{3}$ are given as

$$
V=\frac{\pi r_{1} L}{3}\left(k^{2}+k+1\right)
$$

The volume of liquid removed from the bellows is given by Equation (1), and the relationship between the volume $V \mathrm{~mm}^{3}$ and the curvature radius $R \mathrm{~mm}$ is given as

$$
V=\frac{\pi}{3}\left[-\left\{2 R^{2}+\left(\frac{D}{2}\right)^{2}\right\} t+R\left(\frac{D}{2}\right)\right]
$$

where $D \mathrm{~mm}$ is the lens diameter and $t \mathrm{~mm}$ is the dimension from the lens center to the circumference. The focal length $f \mathrm{~mm}$ from the radius of curvature $R \mathrm{~mm}$ and the refractive index $n$ of the spherical surface are given as

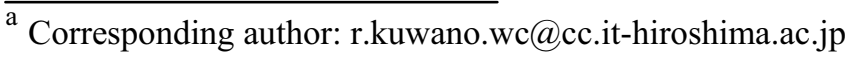




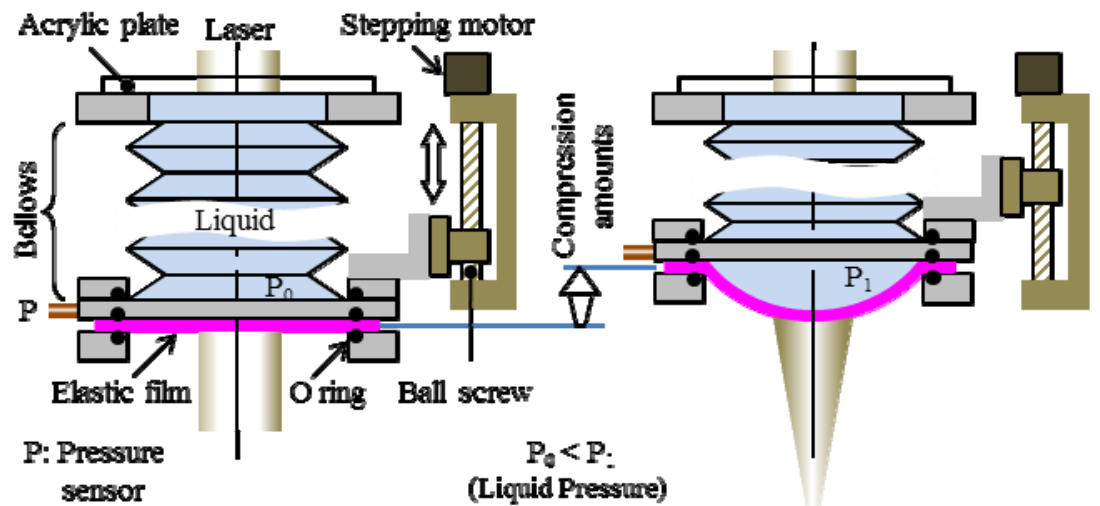

(a) Initial state (b) Convex lens

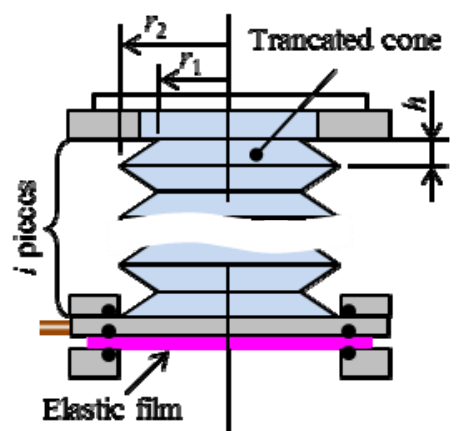

(c) Model of analysis

Figure 1. Principle of the enclosed variable focusing lens.

$$
f=\frac{n-1}{R}
$$

The focal length $f \mathrm{~mm}$ can therefore be calculated from Equations (1) - (3).

\section{Experimental results}

To examine the variable focus function of the prototype lens, the focal length was measured using a laser diode (LD) as a light source. The prototype had a variable focus range of $100-1,000 \mathrm{~mm}$ and a diameter of $65 \mathrm{~mm}$. The focal position was determined by measurement to identify the position of maximum laser power density. Figure 2 shows focal length as a function of the amount of bellows compression. As compression increased from 1 to $12 \mathrm{~mm}$, the focal length changed from 700 to 200 $\mathrm{mm}$. The tendencies of the change in the focal length and in the corresponding model and theoretical values were similar. The actual measurement values were greater due to a lack of liquid movement in the lens portion when liquid pressure was applied. Thus, the focal length increased with the curvature radius of the elastic membrane (i.e., the refractive surface).

Intensity distribution due to focal position changes was measured using a beam profiler (Ophir SP503U). Figure 3 shows intensity distribution measurements with changes in focal length. The beam diameter increased from 0.28 to $0.84 \mathrm{~mm}$ when the focal length increased. The peak value of the laser output decreased from 234 to $137 \mathrm{~mW}$ as the focal length increased. Figure 4 shows that focal length and condensing diameter were proportional, and that they exhibited optical characteristics similar to those of commercially available plano-convex lenses.

To verify applicability for laser welding with large focal lengths, the proposed lens was used in lap welding with a YAG laser (wavelength: 1,064 nm) for mild steel

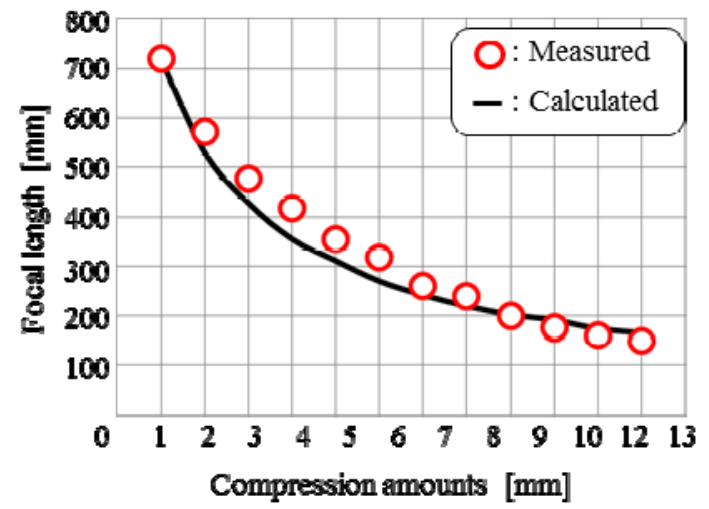

Figure 2. Relationship between compression and focal length.

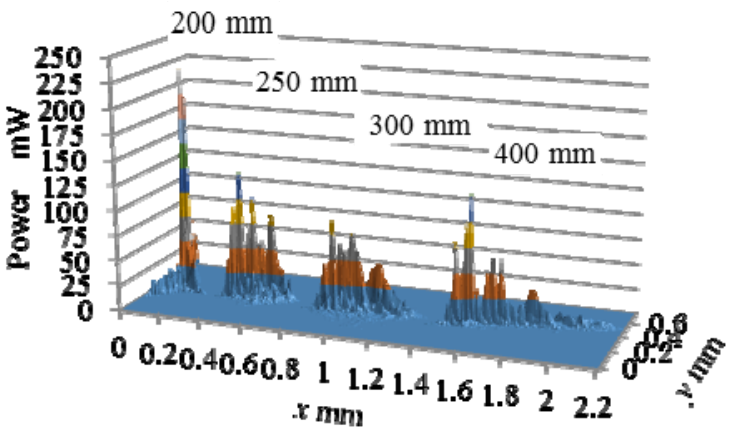

Figure 3. Intensity distribution with different compression amounts.

plate with a thickness of $1 \mathrm{~mm}$. The laser irradiation involved a pulse energy of $47.0 \mathrm{~J}$, an irradiation time of $10 \mathrm{~ms}$, and focal point variation in the range of $200-500$ $\mathrm{mm}$. In the irradiation experiment, the proposed lens and a commercially available plano-convex lens (diameter: 25 $\mathrm{mm}$; focal length: 300,400 and $500 \mathrm{~mm}$ ) were used for 
comparison. Figures 5 (a) and (b) show top view and cross-section representations of the zone welded with a focal length of $400 \mathrm{~mm}$. Characteristics such as the molten zone, the heat-affected zone and the metallic structure were similar. Figure 5 (c) also shows the diameter and penetration depth of the processing zone. In the region where the focal length was large, the lens condensation diameter was greater than that of the commercially available plano-convex lens and the penetration depth decreased. This is attributed to laser scattering caused by the surface roughness of the elastic film used. For focal lengths below $500 \mathrm{~mm}$, the laser processing characteristics of the proposed lens and the plano-convex lens were similar. The durability of the variable focusing lens was verified based on over 3,000 laser irradiations.

\section{Conclusion}

The authors have proposed a variable focusing lens with a bellows structure that allows hydraulic variation of the focal length with a constant amount of liquid in the lens cavity. The lens function and the driving unit used to expand and contract the bellows are integrated. The optical characteristics of the lens were found to be comparable to those of commercially available lenses for focal lengths under $500 \mathrm{~mm}$, and durability was experimentally verified. In future work, an optical element will be applied for the use of high-power fiber lasers in remote welding. To fully elucidate the focal characteristics of the proposed lens, the deformation and surface roughness of the refracting surface material (polymer film) also need to be examined.

\section{References}

1. R. Kuwano, T. Tokunaga : Optics Japan'97, (1997) 85 (in Japanese).

2. R. Kuwano, T. Tokunaga, Y. Otani and N. Umeda : Opt. Rev. 12, 5 (2005) 405.

3. M. C. King and D. H. Berry : Appl. Opt. 9, (1970)2035.

4. S. Sato : Jpn. J. Appl. Phys. 18(1979) 1679.

5. N. Sugiura and S. Morita : Appl. Opt. 32, 22 (1993) 4181.

6. T. Kaneko, I. Kanayama, T. Itogaki, T.Hattori : JSPE semestrial meeting spring, 3 (1996) (in Japanese).

7. D. Y. Zhang, V. Lien, Y. Berdichevsky, J. Choi and Y. H. Lo : Appl. Phys. Lett, 82, 19(2003) 3171.

8. B. H. W. Hendriks, S. Kuiper, M. A. J. van As, and C. J. Renders : Opt. Rev. 12, 3 (2005) 255.

9. M. F. Zaeh, J. Moesl, F. Oefele : Physics Procedia 5 (2010) 19.

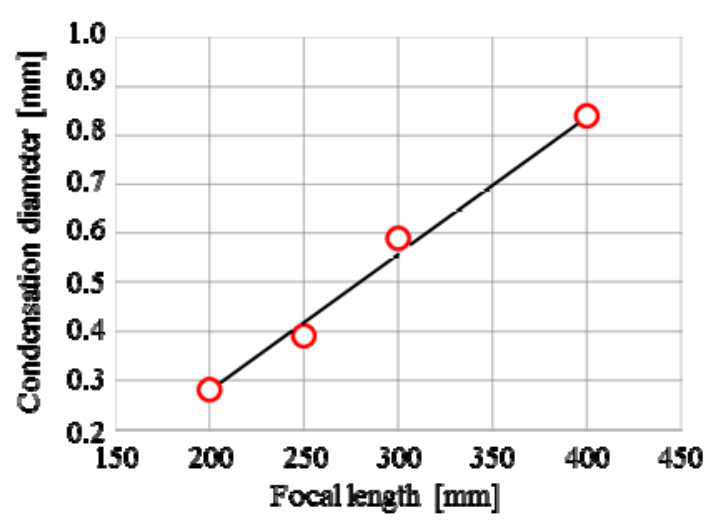

Figure 4. Condensation diameter for different focal lengths.

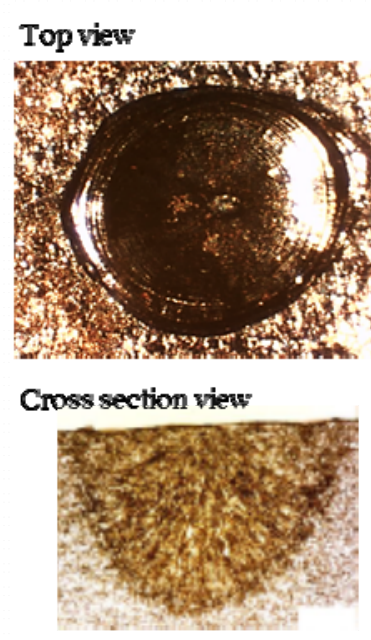

(a) variable focusing lens
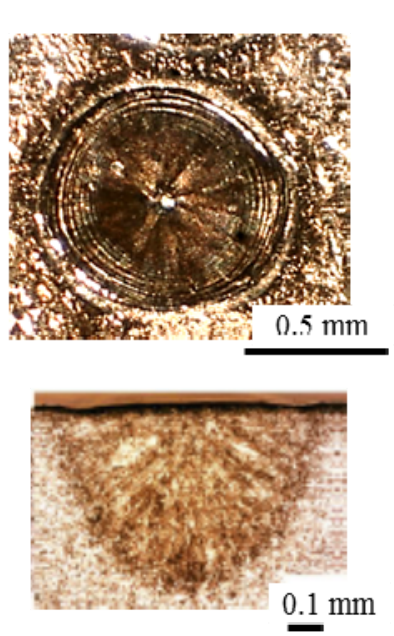

(b) Plano-convex lens

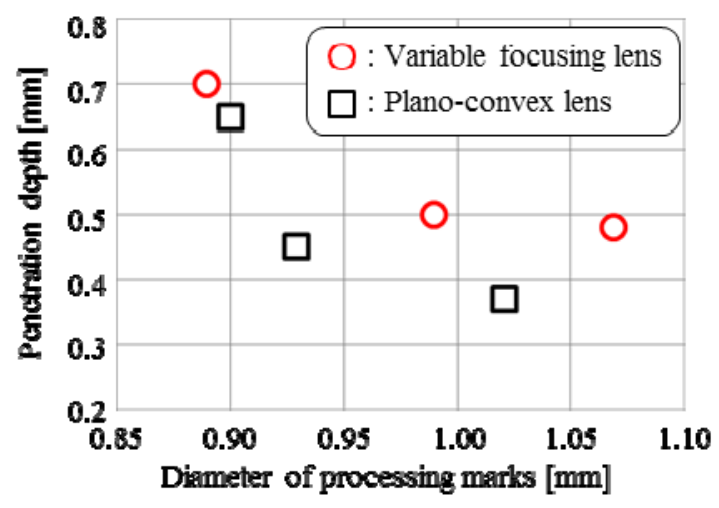

(c) Diameter and penetration depth of the processing zone

Figure 5. Processing-characteristic comparison between a variable focusing lens and a plano-convex lens. 